\section{AB1325 THE FREQUENCY OF ANTI-DFS70 AUTOANTIBODIES IN JAPANESE WOMEN WITH PERI- AND POST- MENOPAUSAL ARTHRALGIA}

K. Miyachi ${ }^{1}{ }^{1,2}$, A. Ihar ${ }^{3}$, B. Sasse ${ }^{4}$, M.Y. Choi ${ }^{5}$, M.J. Fritzler ${ }^{5}{ }^{1}$ Rheumatology and Women's Health, Keigu Clinic; ${ }^{2}$ The Technological Development, Health Sciences Research Institute; ${ }^{3}$ Rheumatology, Keigu Clinic, Yokohama, Japan;

${ }^{4}$ Rheumatology, Monash Health, Victoria, Australia; ${ }^{5}$ Cumming School of Medicine, University of Calgary, Calgary, Canada

Background: The antinuclear antibody (ANA) test is often used as a screening test to aid in the diagnosis of systemic lupus erythematous (SLE and other systemic autoimmune rheumatic diseases (SARD). However, recently it was reported that a certain autoantibody referred to as anti-dense fine speckled 70 (DFS70) are much more common (up to $25 \%$ ) of non-SARD and healthy individuals but rare $<5 \%$ ) in SLE and certain SARD. The frequency of anti-DFS70 is even lower (i.e. $<1 \%$ ) if monospecific anti-DFS70 antibodies are found. Some reports suggested that anti-DFFS70 are more common in younger females, suggesting that hormonal factors may be responsible for the cognate $B$ cell response.

Objectives: The primary objective of our study was to determine the frequency of ant-DFS70 in menopausal women who were referred for evaluation of undifferentiated arthritis (UA).

Methods: 282 women including 105 with UA and an age range of 27 91 years (mean=60.3) were enrolled. Menopausal women were divided into pre-, peri-, and post-menopausal stages according to menstrual regularity. (E2), Follicle stimulating hormone (FSH), rheumatoid factor (RF), ANA (by indirect immunofluorescence (IIF) (MBL) anti-cyclic citrullinated peptide antibody (ACPA: MBL ELISA) and C-reactive protein (CRP) were included in the serology workup. Postmenopausal arthralgia was designated PoMA and perimenopausal arthralgia PeMA.

Results: In PoMA women who received HRT for two months estradiol levels increased, FSH levels decreased and the joint pain visual analogue scale was reduced by $70 \%$, as compared to baseline. Similarly in PeMA women, administration of $600 \mathrm{mg}$ tocopherol $\mathrm{N}$ daily had the same efficacy as that observed in PoMA. The overall frequency of anti-DFS70 of $26.7 \%(28 / 105)$ in PoMA and PeMA women was significantly higher than that in UA females who were diagnosed with rheumatoid arthritis (RA) $(7 / 65: 10.8 \%)$ or primary Sjogren's syndrome $(\mathrm{SjS})(3 / 31: 9.7 \%)(p<0.05)$. In addition, anti-DFS70 Ab was observed primarily in low titer $(1: 40$ - 1:160) ANA positive sera. On the other hand, higher titer ANAs (titer $>1 / 320$ ) were observed in the females that were diagnosed as systemic lupus erythematosus (SLE) $(30 \%)$ or primary SjS $(48.4 \%)$, the majority of whom had lower titers of anti-DFS70, although several sera contained both high titer ANA and anti-DFS70 (see figure)

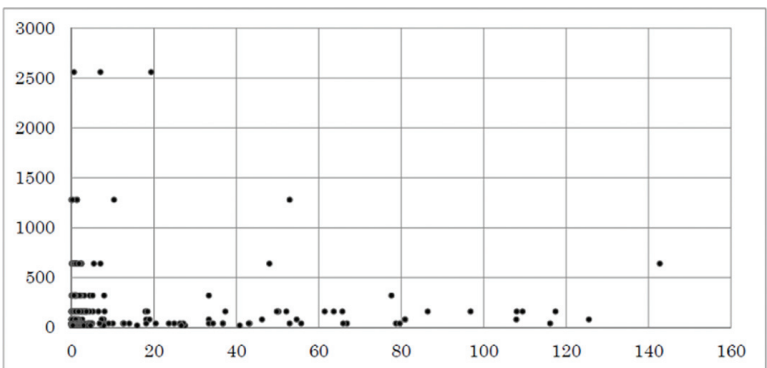

Abstract AB1325 - Figure 1. Relationship of ANA titers and anti-DFS70 levels in peri- and post-menopausal women The majority of anti-DFS70 positive sera had only low to modest ANA titers. ANA titer in vertical axis and anti-DFS70 titers by ELISA on the horizontal axis

Conclusions: Anti-DFS70 was found in higher frequency in PeMA and PoMA women than in women who developed a defined systemic autoimmune rheumatic disease such as SLE, SjS or RA. This is the first study to suggest that the presence of this autoantibody may reflect oestrogen fluctuations or deficiency. A negative association between ANA titer and anti-DFS70 levels by ELISA remains to be confirmed in larger studies.

\section{REFERENCES:}

[1] M. Mahler, P. L. Meroni, L. E. Andrade, M. Khamashta, N. Bizzaro, C. A. Casiano, and M. J. Fritzler. Towards a better understanding of the clinical association of anti-DFS70 autoantibodies. Autoimmun. Rev. 15:198-201, 2016.

[2] K. Conrad, N. Rober, L. E. Andrade, and M. Mahler. The Clinical Relevance of Anti-DFS70 Autoantibodies. Clin. Rev. Allergy Immunol. 52:202216, 2016.
Disclosure of Interest: K. Miyachi Shareholder of: none, Grant/research support from: none, Consultant for: none, A. Ihar: None declared, B. Sasse: None declared, M. Y. Choi: None declared, M. J. Fritzler: None declared DOI: 10.1136/annrheumdis-2018-eular.2013

\section{AB1326 1 NICOTINAMIDE PHOSPHORIBOSYLTRANSFERASE MAY BE NEW FACTORS CONTRIBUTING TO OSTEOARTHRITIS}

L. Sivordova ${ }^{1}$, J. Polyakova ${ }^{1}$, Y. Akhverdyan ${ }^{1}$, V. Kravtsovv ${ }^{1}$, N. Fofanova ${ }^{2}$ B. Zavodovsky'. ${ }^{1}$ Federal State Budgetary Institution "Research Institute of Clinical and Experimental Rheumatology named after A.B. Zborovsky"; ${ }^{2}$ Federal State Budgetary Volgograd Medical University, Volgograd, Russian Federation

Background: Obesity is a condition that prolongs chronic inflammation and promotes synthesis and secretion of pro-inflammatory factors by adipose tissue including adipokines ${ }^{1}$ and can be one of the significant factors in the progression of osteoarthritis (OA) ${ }^{2}$ Nowadays many studies have shown that level nicotinamide phosphoribosyltransferase (Nampt) increases in obesity and can also affect the inflammation in local tissues and together with other adipokines influences the development of $\mathrm{OA}^{3}$

Objectives: To study the effect of weight loss over $5 \mathrm{~kg}$ on the clinical manifestations of $\mathrm{OA}$, indicators of water, lipid metabolism and Nampt serum levels in patients with $O A$.

Methods: We observed 110 people: 80 patients with $O A$ and 30 healthy individu als in the control group with body mass index (BMI) of 25 to $35 \mathrm{~kg} / \mathrm{m}^{2}$, aged 18 to 79 years participated in the study.

Nampt level in serum was determined by indirect solid phase ELISA using a com mercial test systems (RaiBiotech, cat № EIA - VIS -1).

Results: As overweight patients were recruited in the study, hypocaloric diet low in animal fats and physiotherapy has been recommended to all participants. The first group consisted of patients who were able to reduce body weight by $5 \mathrm{~kg}$ and more (18 pers.), the second group - patients with weight reduction of less than $5 \mathrm{~kg}$ and patients without any weight loss (62 pers.). Nampt concentration were evaluated in these patients I groups 4,33 $\pm 0,39$ before treatment to $2,40 \pm 0,23 \mathrm{ng}$ $\mathrm{ml}(\mathrm{t}=5,85, \mathrm{p}<0,001)$. In analysing the parameters before and after treatment, it should be noted that we observed significant decrease in the severity of the clinical manifestations of OA, visfatin level, CRP, and glucose levels and lipid profile in the 1 st group of patients. These data proves that obesity may be an important risk factor for OA progression. As a result, weight loss results in decreasing metabolic disorder severity. In the second group of patients we have seen a decrease in all the parameters, but a significant difference has been observed only in the level of CRP, level of pain at rest and during walking according to VAS scale and total index on the WOMAC

Conclusions: As a result of our study patients with OA with weight loss of more than $5 \mathrm{~kg}$ had more obvious pain relief than patients with the original weight. At the same time a significant improvement has been seen in carbohydrate and lipid metabolism. These findings suggest that there is a possible role of Nampt in the pathogenesis of $O A$.

\section{REFERENCES}

[1] Akhverdyan Y, Zavodovsky B, Seewordova L, et al. Adipokines as new laboratory markers in osteoarthritis. Ann Rheum Dis 2013;72 (Suppls3):702.

[2] Polyakova Y, Seewordova L, Akhverdyan Y. et al. Some pathophysiological aspects of pain syndrome in osteoarthritis. Osteoporosis International 2017;28(Suppl 1:154

[3] . Sivordova LE, Simakova ES, Polyakova JV, et al. Overweight and high level of nicotinamide phosphoribosyltransferase as factors contributing to osteoarthritis progression and metabolic syndrome development. Bulgarian medicine 2016;Tom VI, 1:11-13.

Disclosure of Interest: None declared DOI: 10.1136/annrheumdis-2018-eular.3064

\section{AB1327 CARDIAC MANIFESTATIONS IN SPONDYLOARTHRITIS: PREVALENCE AND PREDICTIVE FACTORS}

J. Eddarami, L. Ichchou. Department of Rheumatology, Mohamed VI University Hospital, Mohammed I University, Oujda, Morocco

Background: Cardiac involvement is a well-recognised complication of spondy loarthritis ( $\mathrm{SpA})$. The spectrum of this condition is large and includes mitral valve disease, conduction disorders and pericarditis, but the aortic disease remains the most characteristic lesion. 
Objectives: The aim of this study is to determine the prevalence of cardiac manifestations and to assess their predictive factors in a population of Moroccan patients suffering from SpA

Methods: We have conducted a cross-sectional study over two months in our department of rheumatology. All SpA patients fulfilled ASAS 2009 criteria. They all have had a cardiac check up with research of clinical cardiac manifestation, 12lead electrocardiogram and trans-thoracic echocardiography.

Data analysis was carried out using the SPSS Statistics 20 software. A univaried analysis as well as multivariate regressions were carried out to identify the factors associated with cardiac manifestations.

Results: We have included 61 men and 31 women with a mean age of 37.34 \pm 12.77 years old. The mean disease duration was $10.59 \pm 7.63$. The median CRP was $9.60 \mathrm{mg} / \mathrm{L}$ (IQR 0-180), the mean ASDAS CRP $2.24 \pm 1.30$ and the mean BASDAI $2.7 \pm 1.99$

Traditional cardiovascular risk factors in our series included dyslipedimia in 15 patients $(18.3 \%)$, hypertension in 10 patients $(10.9 \%)$ and type 2 diabetes in 7 patients $(7.6 \%)$. Mean BMI was $23.88 \pm 5.83 \mathrm{Kgm}^{-2}$. Twenty nine patients $(31.9 \%)$ were overweight and 10 patients (11\%) were obese. Eight patients $(8.7 \%)$ smoked and 3 patients (3.3\%) used alcohol whereas 19 patients $(20.7 \%)$ had a history of smoking and 6 patients $(6.5 \%)$ had a history of alcohol use.

Cardiac manifestations were found in 12 patients (13.6\%): $3(3.3 \%)$ had aortic regurgitation (AR), $1(1.1 \%)$ had aortic dilatation, $1(1.1 \%)$ had aortic thickening, 2 $(2.2 \%)$ had mitral thickening, $1(1.1 \%)$ had mitral regurgitation (MR), $1(1.1 \%)$ had mitral stenosis (MS), 3 (3.4) had pericarditis and $2(2.2 \%)$ had bundle branch block.

In comparison with the group without cardiac manifestations, axial involvement, current enthesitis and extra-articular manifestations were common in patients with cardiac involvement. Furthermore, uveitis was frequent in patients with aortic dilatation and MR. Patients with AR and MS had higher ESR and disease activity compared to the group without heart disease. Patients with pericarditis had an accelerated ESR unlike the group without cardiac involvement.

In univariate analysis, AR was significantly associated with ESR and ASDAS VS $(\mathrm{OR}=1.03$ [1.003-1.063], $\mathrm{p}=0.028 ; 2.75$ [1.062-7.123], $\mathrm{p}=0.037$, respectively). Pericarditis was significantly associated with ESR (OR=1.03 [1.003-1.062], $\mathrm{p}=0.029$ ).

In multivariate analysis, cardiac manifestations were significantly associated with current enthesitis and extra-articular manifestations (OR=4.48 [1.078-18.683], $\mathrm{p}=0.039$ and 6.35 [1.156-34.893], $\mathrm{p}=0.033$, respectively).

Conclusions: Heart disease was common in our study and was associated with the enthesitis involvement and the severity of SpA.

\section{REFERENCES:}

[1] Ozkan Y. Cardiac involvement in ankylosing spondylitis. J Clin Med Res 2016;8:427-30.

[2] Lianne S, et al. Axial spondyloarthritis: the heart of the matter. Clin Rheumatol 2015;34: 995-8.

[3] Pereira IA, et al. Extra-articular manifestations in spondyloarthritis are common and should be screened. Rheumatol Curr Res 2012;2:111.

Disclosure of Interest: None declared

DOI: 10.1136/annrheumdis-2018-eular.6735

\section{AB1328 1 FLARES IN SPONDYLOARTHRITIS: PREVALENCE AND RELATED FACTORS}

J. Eddarami, L. Ichchou. Department of Rheumatology, Mohamed VI University Hospital, Mohammed I University, Oujda, Morocco

Background: Spondylaoarthritis is a chronic condition characterised by alternated periods of flares and stable disease.

Objectives: The aim of this study is to assess the prevalence of patient-reported flares and the validity of this concept through its related factors.

Methods: We have conducted a cross-sectional study over two months in our department of rheumatology. All SpA patients fulfilled ASAS 2009 criteria.

Current and past flares over the last 3 months were recorded. Disease activity and functional status were assessed by the BASDAI score (0-10) and the Bath Ankylosing Spondylitis Functional Index (BASFI; 0-100), respectively. Data analysis was carried out using the SPSS Statistics 20 software. Univaried and multivariate regressions were performed to identify the factors associated with current flare. Results: We included ninety tow patients with SpA, 61 (66.3\%) were men. The mean age was $37.34 \pm 12.77$ years old. The mean disease duration was 10.59 \pm 7.63 year. The mean BASDAI was $2.7 \pm 1.99$ and the median BASFI was 14 (IQR 0-100). Thirty-five patients $(38 \%)$ received antitumor necrosis factor alpha (anti-TNF- $\alpha$ ). Twenty-nine patients (31.5\%) were currently in flare, and 6 patients $(6.5 \%)$ reported only past flares.

In univariate analysis, patients reporting current flares had a current enthesitis $(O R=3.14$ [1.250-7.923], $p=0.015)$ and higher values of BASDAI, ASDAS VS and
ASDAS CRP (OR=2.17 [1.085-4.362], $p=0.029 ; 2.20$ [1.448-3.359], $p=0.0001$; 2.23 [1.495-3.340], $p=2.235$, respectively). These patients had also more extraarticular manifestations (OR=3.37 [1.326-8.601], $p=0.011)$.

In multivariate analysis, the following factors were associated with current flare: higher BASFI (OR=1.05 [1.013-1.098], $p=0.010)$, protein c-reactive $(O R=1.087$ [1.016-1.163], $p=0.016)$, ASDAS VS (OR=10.99 [1.921-62.873], $p=0.007)$, current enthesitis $(O R=25.00$ [2.185-286.146], $p=0.01)$, the presence of extra-articular manifestations $(O R=30.66$ [1.709-550.049], $p=0.02)$ and the use of non steroidal anti-inflammatory drugs during the last 3 months $(\mathrm{OR}=125.064$ [3.334491.0], $\mathrm{p}=0.009$ ).

Conclusions: Our study shows that assessment of flares may provide a good estimation of disease activity.

\section{REFERENCES}

[1] Jacquemin C, et al. Patient-reported Flares in Ankylosing Spondylitis: A Cross-sectional Analysis of 234 Patients. J Rheumatol 2017;44:425-30.

[2] Cooksey $R$, et al. Severe flare as a predictor of poor outcome in ankylos ing spondylitis: a cohort study using questionnaire and routine data linkage. Rheumatology 2015;54:1563-72.

[3] Stone MA, et al. Assessment of the impact of flares in ankylosing spondylitis disease activity using the Flare Illustration. Rheumatology 2008:47:1213-8.

Disclosure of Interest: None declared

DOI: 10.1136/annrheumdis-2018-eular.6806

\section{AB1329 BIOLOGIC AGENTS AND RISK OF SERIOUS INFECTIONS IN RHEUMATOID ARTHRITIS, PSORIATIC ARTHRITIS AND ANKYLOSING SPONDYLITIS: RESULTS BY INTEGRATING TEN-YEAR (2006-2015) ADMINISTRATIVE DATABASES IN THE ITALIAN REGION OF FRIULI VENEZIA GIULIA}

L. Quartuccio $^{1}$, A. Zabotti ${ }^{1}$, S. Del Zotto ${ }^{2}$, L. Zanier ${ }^{2}$, S. De Vita ${ }^{1}$, F. Valent ${ }^{3}$. ${ }^{1}$ Department of Medical Area, Rheumatology Clinic, Udine Academic Hospital "Santa Maria della Misericordia", ${ }^{2}$ Central Direction of Health of Friuli Venezia Giulia, Epidemiology Service; ${ }^{3}$ Institute of Epidemiology, Academic Hospital "Santa Maria della Misericordia", Udine, Italy

Background: Serious infections are a major concern for patients considering biologic agents for rheumatoid arthritis (RA) or psoriatic arthritis (PsA) or ankylosing spondylitis (AS). Evidence from meta-analyses is consistent with an increased risk of serious infections with biologic disease-modifying antirheumatic drugs (bDMARDs) ${ }^{1}$ however, long-term real-life studies are scarce and controversial.

Objectives: To determine the risk of serious infections (i.e., need of hospitalisation) in patients affected by RA, psoriatic arthritis (PsA) or ankylosing spondylitis (AS), treated by bDMARDs in the Italian region of Friuli Venezia Giulia, approximately 1,200,000 inhabitants.

Methods: The regional health information system was used as the source of data. Administrative data from the hospital discharge records database, the medical prescription database, and the database of exemptions from medical charges were linked at the individual patient level through an anonymous univocal sto chastic key. A cohort of patients diagnosed for RA, PsA or AS from 2006 to 2015 was identified from specific national exemption codes $(006,045,054)$ and followed up to either the end of 2015 , or hospitalisation with the main discharge diagnosis of infection, as ICD-9-CM codes, or death, or change of residency out of the Region. Multivariate Cox regression was used to estimate the hazard ratio (HR) of hospitalisation associated with bDMARDs and adjusting for age, sex, Charlson's comorbidity index, calendar year, prescription of steroids and of csDMARDs. Use of bDMARDs was treated as a time-dependent variable.

Results: Overall, the cohort consisted of 5596 subjects: $3216(57,5 \%)$ with RA $1702(30,4 \%)$ with PsA, and $678(12,1 \%)$ with AS. Of them, 940 patients $(16,8 \%)$ had received at least one prescription of a bDMARDs during the study period. During follow-up, $193(3,4 \%)$ subjects were admitted to the hospital with a primary diagnosis of infection. The most frequent infections were upper and lower respiratory tract infections $(86 / 193,44,5 \%)$, followed by sepsis $(19 / 193,9,8 \%)$, and soft tissue and skin infections (16/193, 8,3\%). After adjusting for the abovementioned variables, when starting a bDMARD, the risk of infections was significantly increased, with an observed increased risk of almost 2-fold by the time of the bDMARD prescription ( $\mathrm{HR} 1.694,95 \% \mathrm{Cl} 1.158-2.478, \mathrm{p}=0,0066$ ). Compared with patients $<40$ years if age, the elderly ( $>/=65$ years) showed a much higher risk of infections ( $\mathrm{HR} 3.919,95 \% \mathrm{Cl} 2.286-6.716, \mathrm{p}<0,0001)$. Analyses stratified by disease indicated that RA, PsA, and AS patients starting biologic medications have increased risk of infection.

Conclusions: By integrating the information coming from ten-year administrative databases, RA, PsA and AS patients starting bDMARDs are at high risk of serious infections. This risk is much higher in the elderly. Upper and lower respiratory tract 